\title{
A fast passive and planar liquid sample micromixer
}

\author{
Jessica Melin, Guillem Giménez, Niclas Roxhed, Wouter van der Wijngaart and Göran \\ Stemme \\ Microsystem Technology, Department of Signals, Sensors and Systems, Royal Institute of \\ Technology, SE 100 44, Stockholm, Sweden
}

Received 4th November 2003, Accepted 10th February 2004

First published as an Advance Article on the web 1st March 2004

\begin{abstract}
A novel microdevice for passively mixing liquid samples based on surface tension and a geometrical mixing chamber is presented. Due to the laminar flow regime on the microscale, mixing becomes difficult if not impossible. We present a micromixer where a constantly changing time dependent flow pattern inside a two sample liquid plug is created as the plug simply passes through the planar mixer chamber. The device requires no actuation during mixing and is fabricated using a single etch process. The effective mixing of two coloured liquid samples is demonstrated.
\end{abstract}

\section{Introduction}

New microtechnologies and components have often been driven by the pharmaceutical industry's demand for high quality medicines produced at a rapid rate and a lower cost. In (bio)chemical and biological applications, miniaturization offers a solution to several challenges including increasing throughput, allowing automation, and decreasing costs by reducing the amount of expensive reagents used. In addition, miniaturization promises higher selectivity, higher yield, fewer byproducts, efficient heat management, and increased process safety. ${ }^{1}$ More specifically, fast and efficient mixing of small volumes of reactants is important in areas such as DNA hybridization, cell activation, enzyme reactions, chemical synthesis and protein folding. ${ }^{2}$ For example, the key steps in chemical synthesis include mixing, heating, and cooling. In large reaction vessels, mixing often creates large concentration gradients resulting in formation of byproducts, which lead to overheated areas (i.e. hot spots). However, micro reaction vessels have a larger surface area to volume ratio and allow more effective heat management. ${ }^{1}$

Mixing can be divided into two steps: 1) a heterogeneous mixture having a finely dispersed structure is obtained; 2) diffusion between adjacent domains leads to a homogeneous mixture at the molecular level. ${ }^{1}$ In macrosystems, propellers, stirrers, etc. are used to achieve mixing. Whereas fluids in the turbulence regime do not stay in the same layer and move in a random manner across the flow path, in microsystems, fluids in channels have a low Reynold's number $(R e)$ and the flow is laminar. Fluid in laminar flow (low $R e$ ) remains in the same layer and only passes across the boundary to neighbouring lamellae through molecular diffusion. ${ }^{1}$ At $R e<15$ in an orifice and $R e<30 L / D_{\mathrm{h}}$ for a short channel (where $L$ is the channel length and $D_{\mathrm{h}}$ is the hydraulic diameter), fluid transitions into the laminar regime and inertial effects become minimal while viscous forces dominate. ${ }^{3}$ Although laminar flow may be advantageous when designing flow control microsystems, it is a cumbersome problem when fast mixing of liquids is desired. Molecular diffusion dominates at these low Reynold's numbers and the diffusion time depends on the channel width. Thereby, channels must either be very long to accommodate the diffusion time or very narrow to decrease diffusion time. Unfortunately, both long and narrow channels also result in large pressure drops. However, chaos arises in nonlinear dynamic systems if at least 3 degrees of freedom are present. ${ }^{4}$ In the case of a 2D steady flow, as commonly occurs in microsystems, a time dependent external perturbation can be introduced or a geometrical perturbation in a 3D flow field resulting in chaotic advection. ${ }^{5}$ Passive particles are advected by a periodic, laminar velocity field and exhibit chaotic trajectories enhancing stretching and folding of the fluid and deforming fluid-fluid boundaries. ${ }^{6}$ A perturbation may include heat convection, differential pressure, ultrasonic piezoelectric actuation, vapour pneumatic power, magnetic actuation, etc. The main goal is to stretch and fold the fluid, thereby increasing interfacial areas resulting in faster diffusion, enhanced mass and heat transfer and increased chemical reactions. ${ }^{1}$

Main mixing strategies in microsystems are chaotic advection and lamination (parallel or serial) where sections of liquid are often redistributed. Micromixers can also be categorized as passive or active. Passive mixers require no energy input except the mechanism used to drive fluid flow at a constant rate while active mixers exert active control over the flow field. While active mixers can be activated on demand, they have disadvantages including being difficult to fabricate, operate, clean, and integrate, while passive mixers are usually more robust, easier to implement and often simply entail pushing the fluid through a certain channel geometry. Manz et al. presented a continuous flow mixer based on separating liquid in narrower channels and reunifying them allowing faster molecular diffusion. ${ }^{7}$ Other passive lamination based mixers where fluid is split in a parallel or serial manner have also been demonstrated by Wurmus et al., ${ }^{8}$ Branebjerg et al. ${ }^{9}$ Larsen et al. ${ }^{10}$ and Jensen et al. ${ }^{11}$ Yet others have created plumes to increase the surface area for mixing. ${ }^{12}$ Active mixers include a simple channel system where side channels introduce pressure perturbations or time-dependent dielectrophoresis forces to induce chaotic advection. ${ }^{4}$ Kim et al. presented a mixer based on joined droplets which are moved around by electrowetting on dielectric and subsequently the interface between liquids is distorted by a velocity gradient in the droplet. ${ }^{13}$ Acoustic transducers have also been used to produce chaotic advection. ${ }^{14}$ Chaotic mixing by a geometrical perturbation has been shown where helical flow is perturbed by hyperbolic points through barriers embedded in a channel ${ }^{14}$ or via a herringbone structure on the bottom of the channel ${ }^{15}$ or a $3 \mathrm{D}$ serpentine channel. ${ }^{16}$ In this work, we present a novel and effective truly planar passive micromixer.

\section{Experimental}

\section{Micromixer design}

The novel micromixer utilizes surface tension effects to create a time dependent flow pattern inside a multi-sample liquid plug as this plug moves (pressure driven) through a geometrical chamber resulting in a fully mixed liquid plug. The basic mixing principle was previously presented at $\mu$ TAS ' $03 .{ }^{17}$ The micromixer is a truly planar device and creates a larger mass transfer inside the liquid plug than most passive devices presented previously where mass 
transfer relative to the samples being mixed is often limited. As can be seen in Fig. 1, the micromixer chamber consists of a main meandering channel, where the channel walls are perforated. These perforations create small connecting channels between the parallel segments of the meandering main channel. Two discrete liquid samples enter the mixing chamber in a laminar manner. When the liquid wets one side of the first perforated wall, liquid enters the perforations but does not exit due to surface tension, see magnification in Fig. 1. However, as the liquid proceeds to the next main channel segment, the other side of the perforated wall becomes wetted. Thereby, the liquid/air interface of the perforation is replaced by a liquid/liquid interface, allowing liquid to flow freely through the perforations. This phenomenon continues for each subsequent main channel segment as the liquid passes through the mixing chamber.

The mixer geometry ensures that as the multi-sample discrete liquid plug travels through the subsequent meandering main channel segments, the flowlines from the receding liquid front (RLF) and advancing liquid front (ALF) constantly change over time. This causes the liquid to be constantly redistributed within the liquid sample plug. In other words, the liquid is folded onto itself because of the constantly changing internal flowlines of the liquid plug. Fig. 2 shows a schematic of the flow pattern in the discrete liquid plug as it proceeds through the mixer chamber.

Seen in a different way, the two-sample liquid plug can be seen as a static droplet where its internal flow pattern changes due to the constantly changing positions of the receding and advancing liquid front as demonstrated in Fig. 3.
Note that the micromixer relies on both liquid samples being spreading wetting liquids to function properly. The sample must also be discrete for the mixer to function i.e. a high surface tension interface between the advancing front of the liquid plug and the preceding medium (i.e. air or potentially oil) as well as between the receding front of the liquid plug and the following medium. Fig. 4 illustrates what was observed when a continuous flow of two samples was injected into the micromixer, i.e. the combined volume of the two liquid samples is greater than the mixing chamber volume. The resulting flow in the mixing chamber is laminar in this case. Therefore, the mixing chamber geometry must be designed with consideration for the volume of sample to be used in the final application.

Note that the structures we tested had a hydrophobic ceiling (PDMS) and hydrophilic floor and walls (native oxide covered silicon). If the entire mixer chamber were hydrophobic, the liquid would not enter the microchannels in the perforated walls. However, one can adapt the geometry of the perforations to allow the mixer to function even if the structure is completely hydrophobic as shown in Fig. 5.

\section{Fabrication}

The micromixer was fabricated by patterning a silicon wafer using photoresist and etching the pattern a depth of $50 \mu \mathrm{m}$ using deep reactive ion etching. The mask layer was then removed and the wafer was cleaned. The wafer was diced into individual chips and each chip was packaged by covering the top surface with a layer of

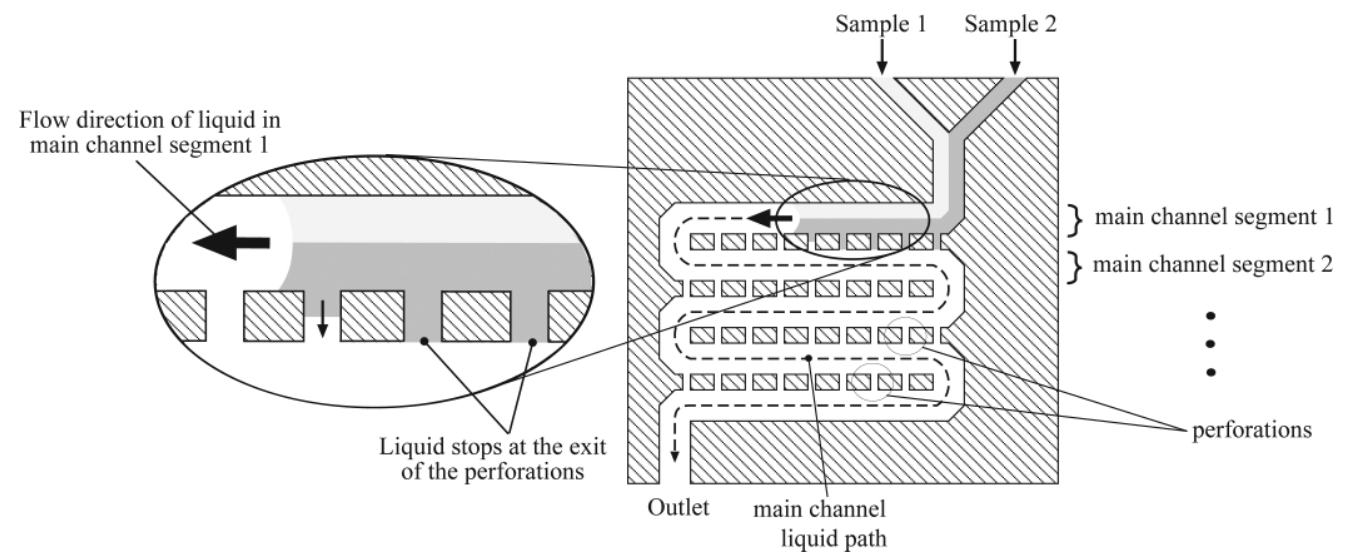

Fig. 1 Top view of the micromixer as two samples enter the first main channel segment. The main channel of the device used in our experiments has a width of $50 \mu \mathrm{m}$, depth of $50 \mu \mathrm{m}$, length of $12 \mathrm{~mm}$, perforated wall thickness of $10 \mu \mathrm{m}$, perforation channel width of $7 \mu \mathrm{m}$, and the mixing chamber volume was $30 \mathrm{~nL}$.

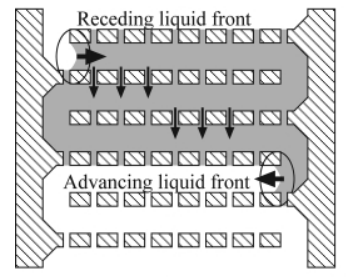

a)

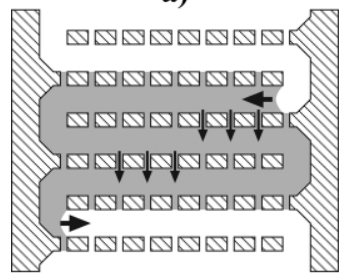

e)

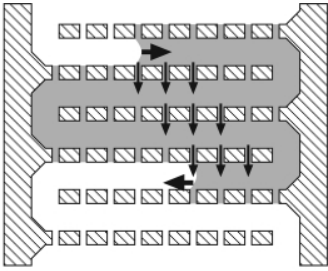

b)

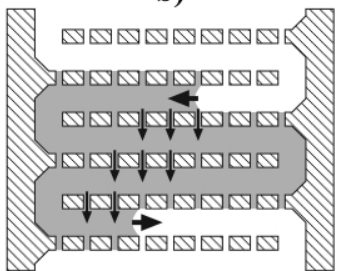

f)

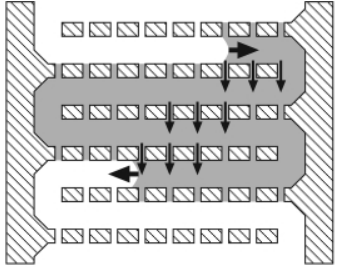

c)

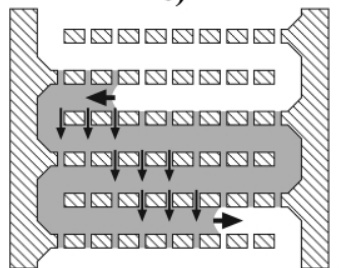

g)

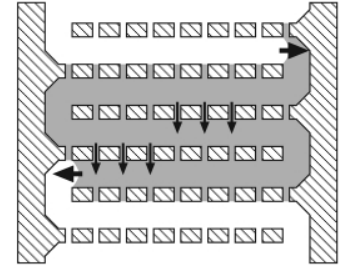

d)

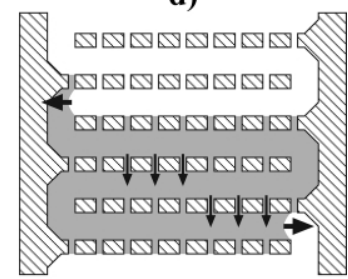

h)

Fig. 2 Schematic showing the time dependent flow pattern inside a liquid plug as it moves through the mixing chamber (frames are ordered consecutively from a) to h)). The black arrows indicate the flow in the liquid plug at a specific moment in time. While the liquid fronts follow the meandering main channel, the liquid flow is 'shorted' through the perforations. The liquid plug is depicted as homogeneous for the sake of clearer visualization of the mixing action. 
polydimethylsiloxane (PDMS), thereby sealing the channel system. Holes were cut through the PDMS with a sharp steel tube at the inlet and outlet ports and syringes inserted and fixed with epoxy for testing purposes. The device and steel connectors were also fixed to a Petri dish with epoxy for increased ease of handling the device during testing. A cross-sectional schematic of the fabricated device is shown in Fig. 6.

\section{Results and discussion}

Both the flow pattern inside the liquid plug and degree of mixing in the micromixer were evaluated. The flow pattern in the mixing chamber was verified by adding a microsphere solution to the mixer and observing the path of these particles through the mixing chamber under the microscope. Common ways of testing the mixing of a micromixer include imaging of fluorescent sample liquids, a $\mathrm{pH}$ colour indicator and a base, or two coloured liquids. The mixing performance was evaluated using dyed water samples, filming the micromixer under an optical microscope, and analyzing the colour of the liquid at the outlet of the micromixer. Mixing evaluation was performed using glycol and ethanol based coloured dyes (yellow and red) in deionized water that were prefiltered using a $0.45 \mu \mathrm{m}$ Millipore filter. A CCD camera was used to film the mixer through a microscope at 25 frames per second. To introduce both liquids simultaneously to the mixing chamber, the syringe was used at the outlet to gently suck the liquids to a conventional onchip Y-junction upstream of the mixing chamber. After both liquids reached the Y-junction, a vacuum pump was used, allowing both liquids to enter the mixing chamber.

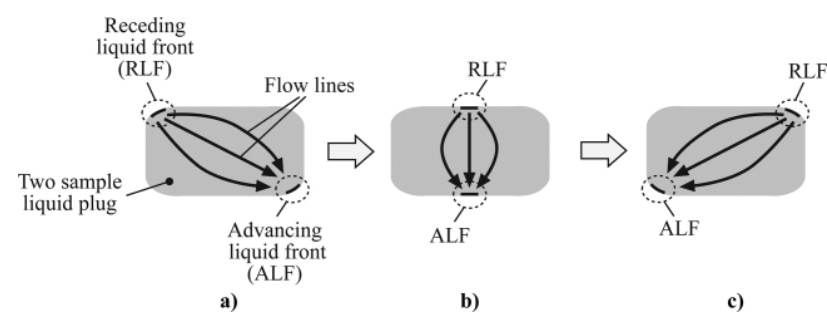

Fig. 3 Schematic of the two liquid samples as a liquid plug, where the flow pattern depends on the position of the plug in the mixing chamber. Frames a), b), and c) correspond roughly to the position of the liquid plug in Fig. 2 a), b), and d).

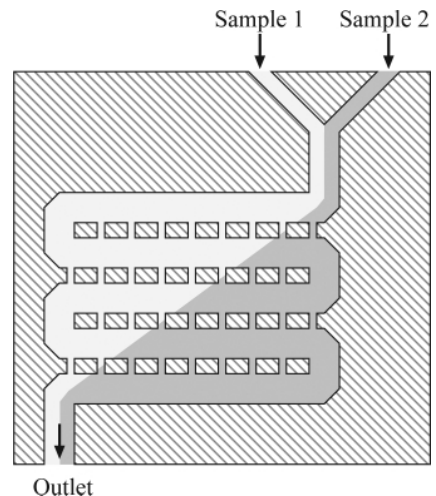

Fig. 4 Schematic of the laminar flow in the mixing chamber if the combined volume of the two samples exceeds the mixing chamber volume, i.e. continuous flow condition.

\section{Flow pattern verification}

A solution of microspheres (Dynabeads, $2.8 \mu \mathrm{m}$ diameter) and deionized water were injected in the micromixer and the flow pattern inside the liquid plug was observed. Fig. 6 shows a close-up of the mixing chamber and the area surrounding one microsphere is highlighted for easier visualization. The curved white line traces the path of that particular particle through the perforations. The sequential video frames in Fig. 7 show the path of the particle flowing through the perforations from the receding front towards the advancing front of the liquid plug.

\section{Colour analysis}

The mixing quality was evaluated by comparing video frames of the liquid at the outlet of the mixer with those of an identical meandering channel structure where the channel walls have no perforations, operated under identical conditions. In this way, we could compare the quality of mixing in the non-perforated structure to the enhanced mixing in the perforated structure. Video frames of the mixer in operation were first calibrated by subtracting the background colour of the silicon device. Fig. 8 shows a photograph of the micromixer and a close-up photograph of a typical area where colour comparisons were performed $(12 \times 24$ pixel area at the device outlet). Note that this particular mixing chamber is diamond shaped instead of rectangular. A rectangular shaped mixing chamber has a flow pattern periodicity equal to one full meander in the mixing chamber whereas a diamond shaped mixing chamber results in an aperiodic flow pattern. This contributes to randomness, thereby enhancing mixing.

Note that it is difficult to achieve a discrete liquid sample of two liquids using the conventional upstream Y-junction. Therefore, a flow-through scenario was evaluated in which the flow lines continuously changed between the mixer chamber inlet (fixed) and advancing liquid front rather than between the receding liquid front and advancing liquid front as the liquid initially enters the mixer chamber. Under these conditions only the initial liquid exiting the mixing chamber is mixed. Fig. 9 shows a schematic of the movement of flowlines during the evaluation of the mixer (compare this to the ideal flowline movement in a discrete liquid plug passing through the mixing chamber in Fig. 3).

The degree of mixing at the outlet was evaluated directly after the advancing front reaches the mixing chamber outlet ( 0.4 seconds after entering the mixing chamber and before the flow pattern becomes laminar, as illustrated in Fig. 4). With this measurement procedure, mixing enhancement in the perforated structure compared to the nonperforated structure was clearly observed. The RGB (red-green-blue) content for each cross-channel pixel (each

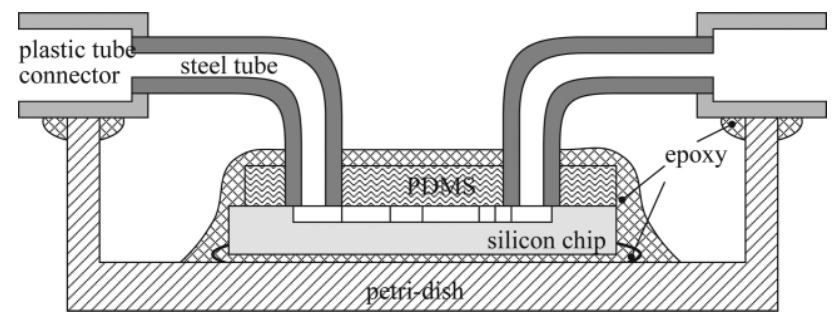

Fig. 6 Schematic of micromixer packaged using a fast and inexpensive method.
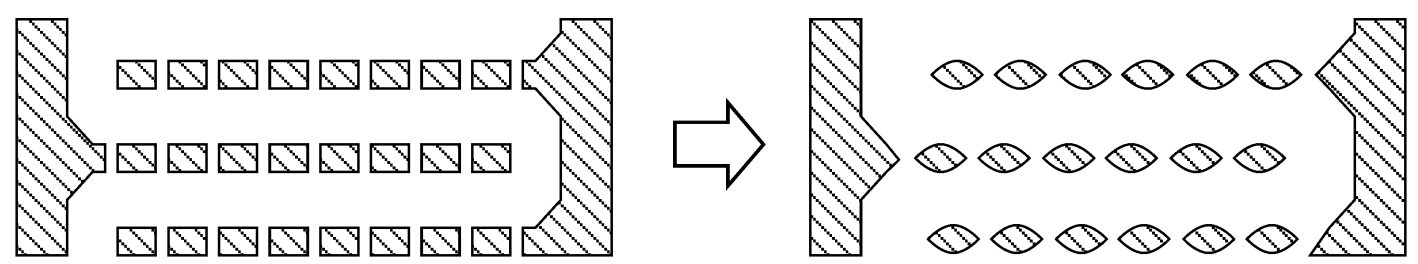

Fig. 5 Perforations resembling microchannels can be used if mixer chamber is hydrophilic (left). Perforation geometry can be redesigned such that completely hydrophobic surfaces can be used in the mixer chamber (right). 
colour value is an average of 24 pixels along the length of the outlet channel) at the outlet of the perforated and non-perforated structures is shown in Fig. 10 and 11. The standard deviation for the $\mathrm{R}, \mathrm{G}$, and B content as well as the average RGB content are much greater for the non-perforated structure than the perforated structure as shown in Fig. 10 and 11. One can clearly observe the even colour distribution in the perforated structure, indicative of a well-mixed sample, and the uneven colour distribution at the outlet of the nonperforated structure. Secondary flows due to the shape of the channel as well as diffusion occur in both the non-perforated structure and perforated structure. As can be seen from Fig. 11, this contributes to minimal mixing compared to the enhanced mixing in the perforated structure. We clearly observed our mixing principle in the perforated chamber (tracing the path of microspheres in the perforated chamber confirm the mixing principle) and assume a linear relationship between the liquid colour intensity and the degree of mixing.

The diffusion time for water in a $50 \mu \mathrm{m}$ wide straight channel is 2.5 seconds according to Fick's Law. The flow rate of the liquid plug through the mixing structure $\left(75 \mathrm{~nL} \mathrm{~s}^{-1}\right)$ was experimentally verified to be fast enough to minimize the mixing effect of pure diffusion, but slow enough to allow visualization with the CCD

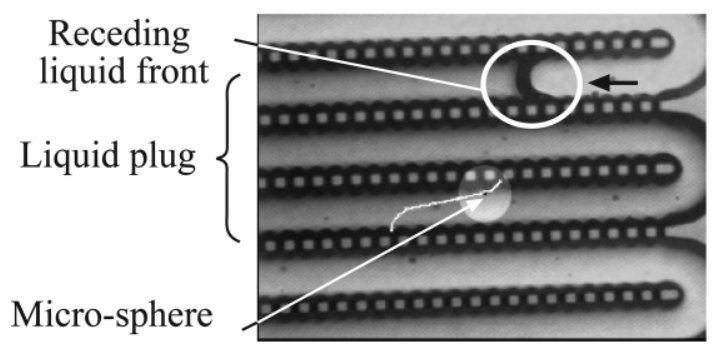

a)

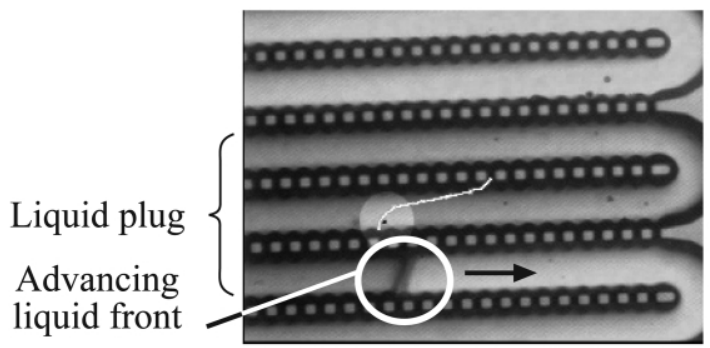

c)

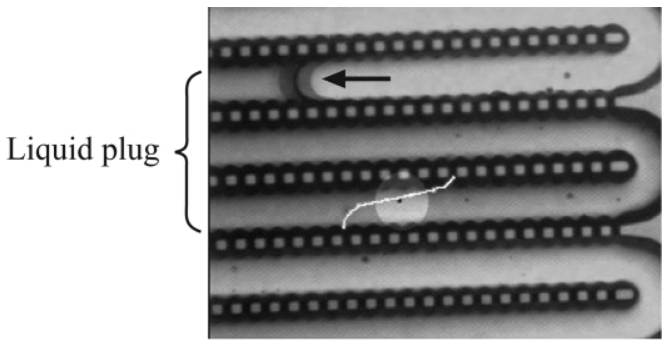

b)

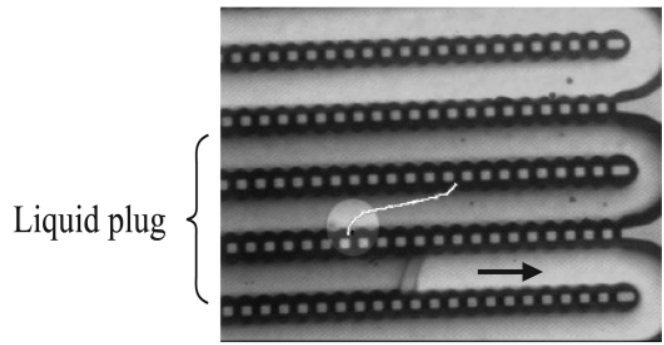

d)

Fig. 7 Video frames showing the path of a microsphere flowing through the perforations from the receding liquid front to the advancing liquid front as the liquid plug proceeds through the mixing chamber a)-d).

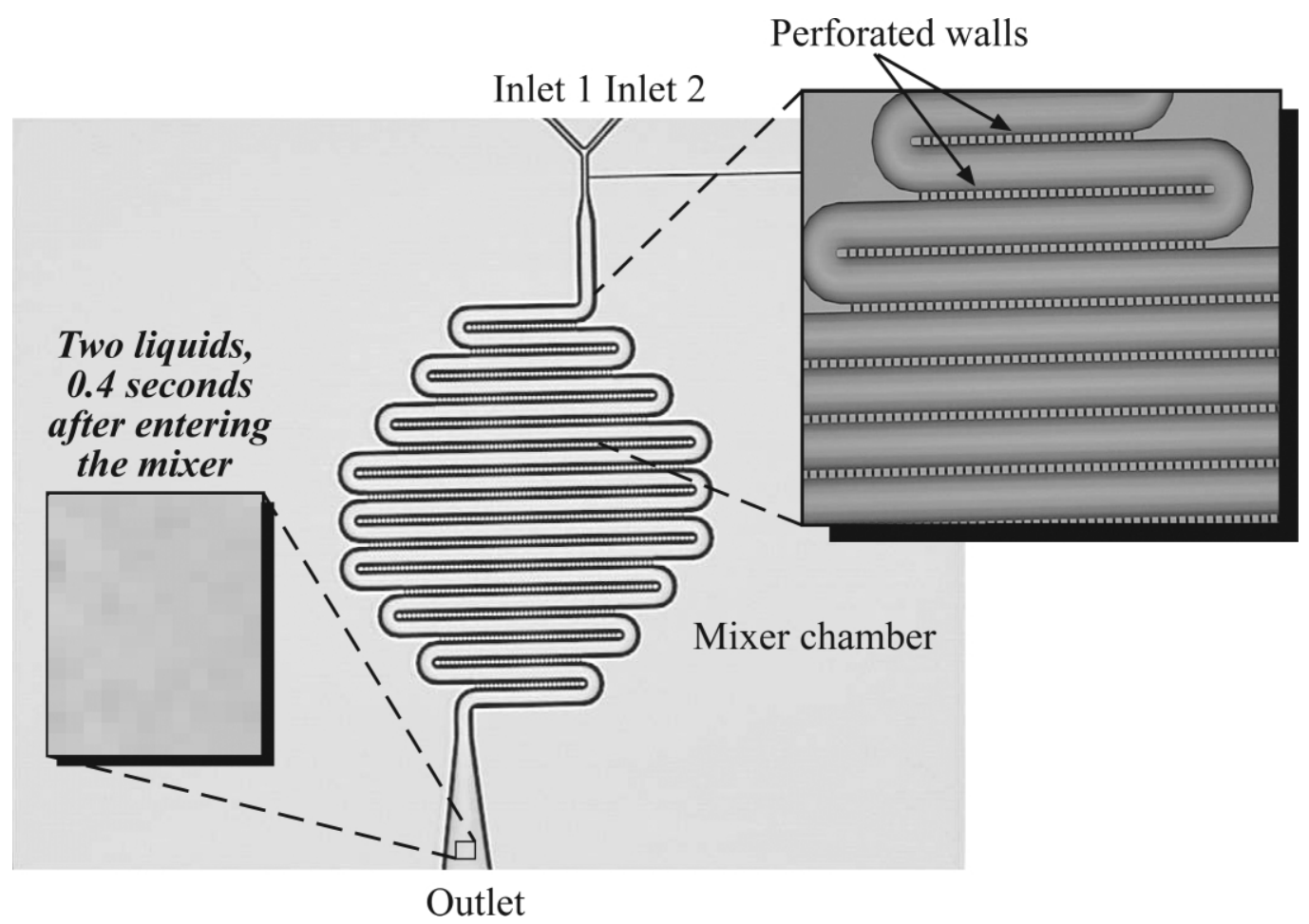

Fig. 8 Photograph of the complete micromixer (main picture), a close-up photograph (top right) and a $12 \times 24$ pixel video frame (left bottom) of a mixed liquid sample at the outlet (showing a nearly homogeneous greyscale intensity). 


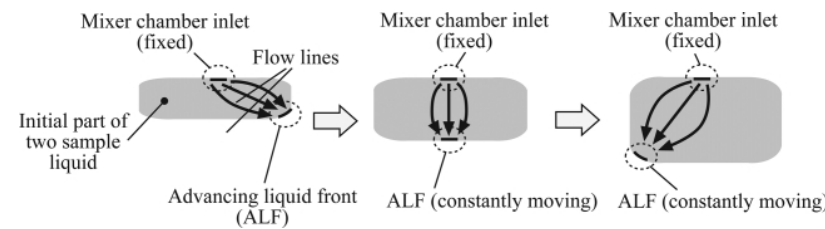

Fig. 9 Schematic showing flowline movement between fixed mixing chamber inlet to constantly moving advancing liquid front as the liquid enters the mixing chamber (as occurs during evaluation of the mixing chamber).
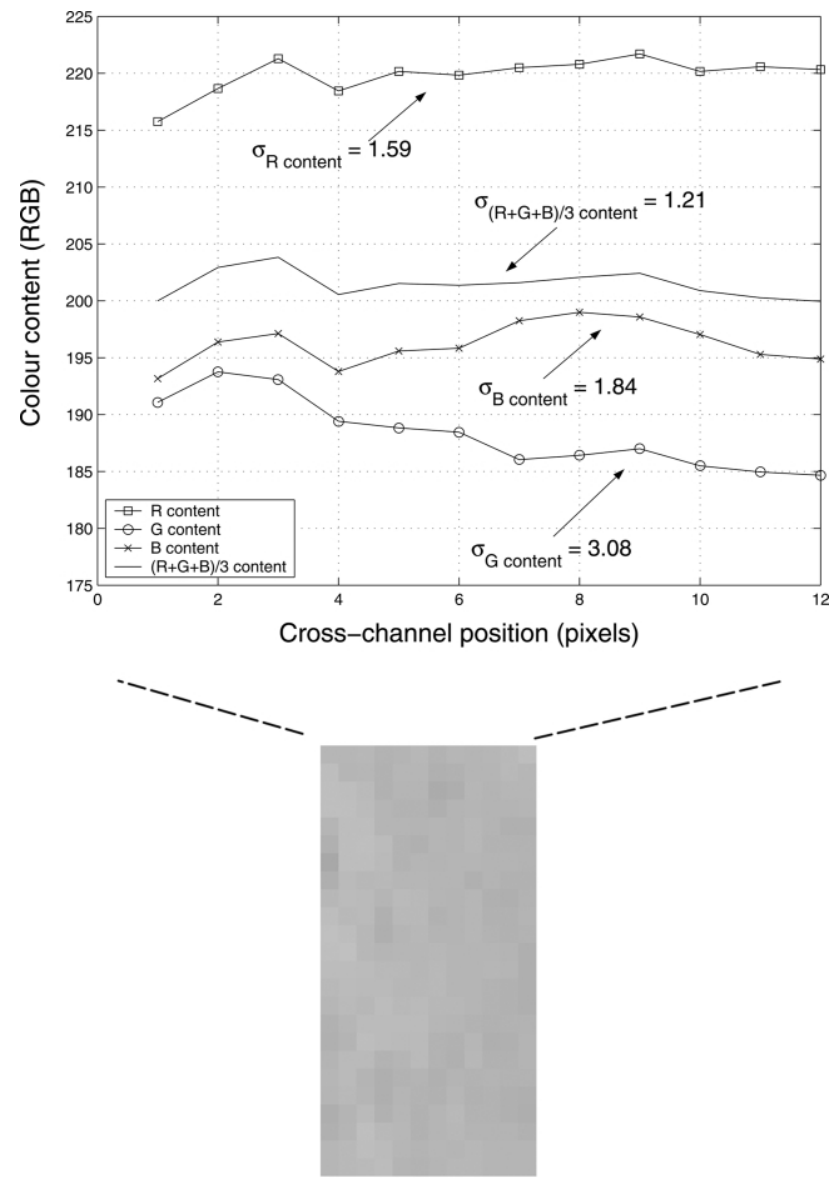

Fig. 10 A $12 \times 24$ pixel video frame of the liquid at the output of the perforated structure (bottom). The corresponding colour profile of the liquid output (top). The average RGB profile and the individual R, G, and B contents are plotted and each corresponding standard deviation $(\sigma)$ is indicated. camera ( 25 frames per second). Note that the Reynold's number of the liquid in the mixing chamber is approximately 0.1 under these conditions.

\section{Integration}

One challenge encountered during testing was timing the entry of the two samples in the mixing chamber. Occasionally one sample enters the chamber prior to the other, forming an air bubble which hinders mixing. This problem can be overcome by replacing a regular Y-junction at the entry by a completely compatible liquidtriggered liquid planar microvalve presented by our group earlier. ${ }^{18}$ As is shown in Fig. 12, the valve acts as a geometrical stop-valve when either sample 1 or sample 2 reaches the junction due to the surface tension/surface energy of the liquid. However, the junction allows liquid to flow through into the mixer when both liquid samples are present at the junction since liquid contact will be made

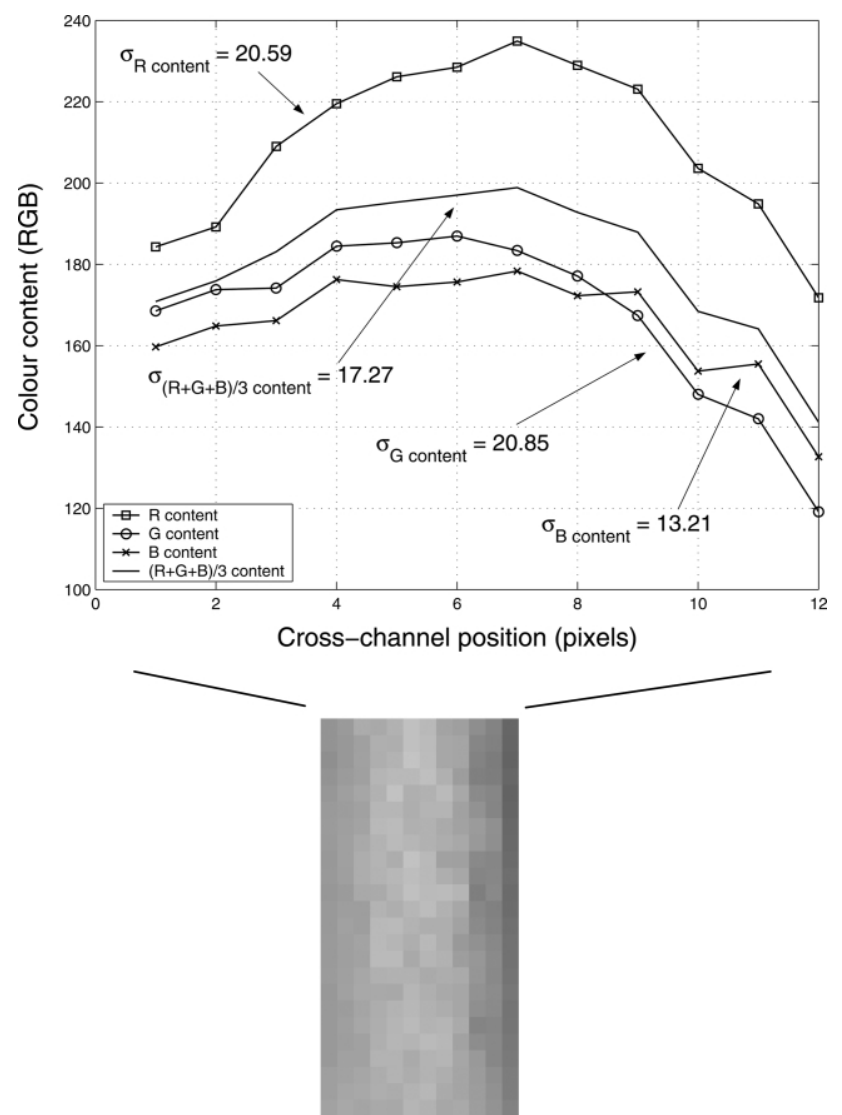

Fig. 11 A $12 \times 24$ pixel video frame of the liquid at the output of the nonperforated structure (bottom). The corresponding colour profile of the liquid output (top). The average RGB profile and the individual R, G, and B contents are plotted and each corresponding standard deviation $(\sigma)$ is indicated.

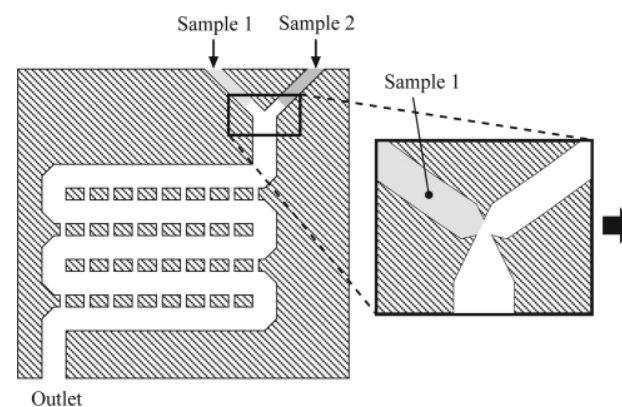

a)

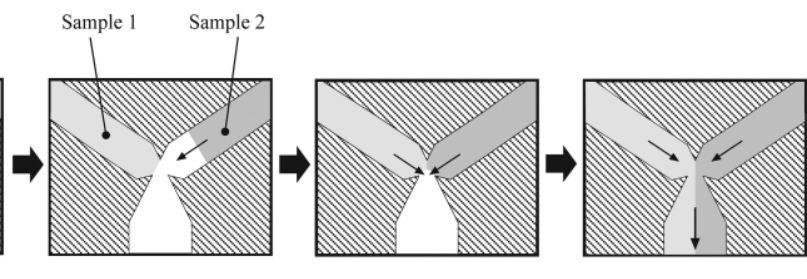

c) d)

Fig. 12 Bubble formation can be avoided by integrating the planar liquid-triggered microvalve. a) Sample enters and stops at the junction of the microvalve due to surface tension, b) sample 2 enters, c) sample 1 and sample 2 make liquid contact at the junction, d) sample 1 and sample 2 proceed to the mixing chamber in a laminar manner. 
at the junction. In other words, if one sample arrives at the junction earlier than the other sample, it will wait to proceed to the mixing chamber until the other sample arrives at the junction.

\section{Conclusions}

The novel passive and planar micromixer creates a constantly changing flow pattern as the liquid samples simply pass through the mixer chamber. The mixer design is based on surface tension and geometry and works for discrete liquid samples. The mixing performance was demonstrated to achieve a very homogeneous mixture after only 0.4 seconds, a four-fold decrease in mixing time compared to diffusion. Note that restrictions of the CCD camera prohibited us from studying the limits of the mixing speed. The device is uncomplicated to fabricate and can be integrated with other useful planar microfluidic components.

\section{References}

1 M. Kakuta, F. G. Bessoth and A. Manz, Microfabricated devices for fluid mixing and their application for chemical synthesis, Chem. Rec., 2001, 1(5), 395-405.

2 R. H. L. Liu, J. Yang, M. Z. Pindera, M. Athavale and P. Grodzinski, Acoustic microstreaming for biological sample mixing enhancement, 2nd Annual International IEEE-EMBS Special Topic Conference on Microtechnologies in Medicine and Biology, Madison, WI, USA, May 2-4, 2002.

3 P. Gravesen, J. Branebjerg and O. S. Jensen, Microfluidics - a review, J. Micromech. Microeng., 1993, 3, 168-182.

4 Y. K. Lee, J. Deval, P. Tabeling and C. M. Ho, Chaotic mixing in electrokinetically and pressure driven micro flows, The $14^{\text {th }}$ IEEE Workshop on MEMS, Interlaken, Switzerland, January, 2001.

5 D. S. Kim, S. W. Lee, T. H. Kwon and S. S. Lee, Barrier embedded chaotic micromixer, IEEE, The Sixteenth Annual International Conference on Micro Electro Mechanical Systems, Kyoto, Japan, January 19-23, 2003.

6 M. A. Stremler, M. G. Olsen, B. H. Jo, R. J. Adrian, H. Aref and D. J. Beebe, Chaotic mixing in microfluidic systems, Solid-State Sensor and Actuator Workshop, Hilton Head Island, SC, USA, June 4-8, 2000.
7 F. G. Bessoth, A. J. deMello and A. Manz, Microstructure for efficient continuous flow mixing, Anal. Commun., 1999, 36, 213-215.

8 N. Schwesinger, T. Frank and H. Wurmus, A modular microfluid system with an integrated micromixer, J. Micromech. Microeng., 1996, 6, 99-102.

9 J. Branebjerg, P. Gravesen, J. P. Krog and C. R. Nielsen, Fast mixing by lamination, MEMS '96, An Investigation of Micro Structures, Sensors, Actuators, Machines, and Systems, San Diego, CA, USA, February $11-15,1996$

10 U. D. Larsen, J. Branebjerg and G. Blankenstein, Proceedings of the 2nd International Symposium on Miniaturized Total Analysis Systems, uTAS96, Basel, Switzerland, November, 1996.

11 T. M. Floyd, M. W. Losey, S. L. Firebaugh, K. F. Jensen and M. A. Schmidt, Microreaction Technology:Industrial Prospects, Springer, Berlin, p. 171.

12 R. Miyake, T. S. J. Lammerink, M. Ewenspoek and J. H. J. Fluitman, Micro mixer with fast diffusion, MEMS '93, An Investigation of Micro Structures, Sensors, Actuators, Machines and Systems, Fort Lauderdale, FL, USA, February 7-10, 1993.

13 J. Fowler, H. Moon and C. J. Kim, Enhancement of mixing by dropletbased microfluidics, MEMS '02, The 15th IEEE International Conference on Micro Electro Mechanical Systems, Las Vegas, Nevada, USA, January 20-24, 2002.

14 H. Jagannathan, G. G. Yaralioglu, A. S. Ergun and B. T. Khuri-Yakub, An implementation of a microfluidic mxer and switch using micromachined acoustic transducers, The $16^{\text {th }}$ Annual International IEEE Conference on Micro Electro Mechanical Systems, Kyoto, Japan, January 19-23, 2003.

15 A. D. Stroock, S. K. W. Dertinger, A. Ajdari, I. Mezic, H. A. Stone and G. W. Whitesides, Chaotic mixer for microchannels, Science, 2002 295, 647-651.

16 R. H. Liu, M. A. Stremler, K. V. Sharp, M. G. Olsen, J. G. Santiago, R. J. Adrian, H. Aref and D. J. Beebe, Passive mixing in a threedimensional serpentine microchannel, J. Microelectromech. Syst., 2000, 9(2), 190-197.

17 J. Melin, G. Gimenez, N. Roxhed, W. van der Wijngaart and G. Stemme, A passive 2-dimensional liquid sample micromixer, Proceedings of $\mu$ TAS '03 Seventh International Conference on Micro Total Analysis Systems, Squaw Valley, CA, USA, October 5-9, 2003.

18 J. Melin, N. Roxhed, G. Gimenez, P. Griss, W. van der Wijngaart and G. Stemme, A liquid-triggered liquid microvalve, Transducers '03 The $12^{\text {th }}$ International Conference on Solid State Sensors, Actuators, and Microsystems, Boston, MA, USA, June 8-12, 2003. 